\title{
Design of a quasi-periodic vibration energy harvester based on an electromagnetic technique
}

\author{
Zakaria Zergoune*, Najib Kacem and Noureddine Bouhaddi \\ University Bourgogne Franche-Comté, FEMTO-ST Institute, CNRS/UFC/ENSMM/UTBM, Department of Applied Mechanics, 25000 \\ Besançon, France
}

\begin{abstract}
In the present paper, a quasi-periodic vibration energy harvester with magnetic coupling is proposed using the benefits of the energy localization. The proposed quasi-periodic system consists of moving magnets held by elastic structures and coupled by a magnetic force. The mistuning of the device can be achieved by changing either the linear mechanical stiffness or the mass. The whole system has been modelled by forced Duffing equations for each degree of freedom, which include the magnetic nonlinearity and the mechanical damping. The governing equations have then been solved using the harmonic balance method coupled with the asymptotic numerical method. The obtained numerical results show that the total harvested power was increased by $11 \%$ with a bandwidth of $2.7 \%$ thanks to the energy localization phenomenon compared to the reference case.
\end{abstract}

\section{INTRODUCTION}

Embedded systems have gained accuracy, reliability, robustness while becoming miniaturized but they are still limited by the life cycle of their power system. Over the last few years, a great interest for scientific research is showing in a promising alternative to ensure the energy autonomy of nomadic devices by scavenging the ambient mechanical energy. This approach has a strong potential for innovation, miniaturization, respect for ecological issues and is part of the theme of renewable energies as well.

In our environment, diverse ambient energy sources are available and their conversion into electrical energy is a major challenge to increase the autonomy of isolated or abandoned systems. Each environment can correspond to one or more energy sources such as light, wind, thermal gradients, mechanical vibrations. For each of these sources, one or more conversion principles exist for generating electricity. Mechanical vibration sources provide potential energy that can be scavenged for charging self-powered systems. In the last decade, several design of energy harvesters, based on different conversion mechanisms, have been proposed [1,2]. Vibration energy harvesters are commonly based on three different conversion techniques, namely piezoelectric $[3,4]$, electromagnetic $[5,6]$, and for MEMS technology electrostatic [7].

The bulk of the VEH devices proposed in the literature have a chief drawback in that they are effective in a restricted frequency bandwidth around their resonance frequency. Several techniques have been reported in order to enhance the energy harvesting performances. Namely, the multimodal methods and nonlinear have been widely studied [8-10] for enlarging the effective frequency bandwidth and giving more reliable results.

The proposed study deals with the design and modelling of a novel quasi-periodic nonlinear VEH under a harmonic base excitation. The designed harvesting devise used the energy localization and the nonlinear vibration to enhance the harvested performance. Elastic suspensions attached to the moving magnets were used to guarantee a perfect vertical displacement and to reduce the mechanical damping as well. The proposed harvester, operating at low frequencies, demonstrated high performances in term of bandwidth and harvested power by combining the benefits of the low mechanical damping, the energy localization phenomenon and the nonlinearity.

\section{SYSTEM MODELING}

In the present section, two middle moving magnets are considered as illustrated in the equivalent mechanical and electrical model (Figure 1). The proposed harvesting devise is modelled using two forced duffing equations. So, the governing equation of the designed harvester can be written as:

$$
m \ddot{x}_{j}+c \dot{x}_{j}+F_{j}^{m e}(x)+F_{j}^{m g}(x)=-m \ddot{Y}
$$

where $j=1,2$ and $c=c_{e}+c_{m}$ with $c_{e}$ and $c_{m}$ stand for the mechanical and electrical damping factors respectively. $F_{j}^{m e}$ and $F_{j}^{m g}$ are respectively the mechanical and magnetic forces for each moving magnet.

* Corresponding author: zergoune.uni@ gmail.com 


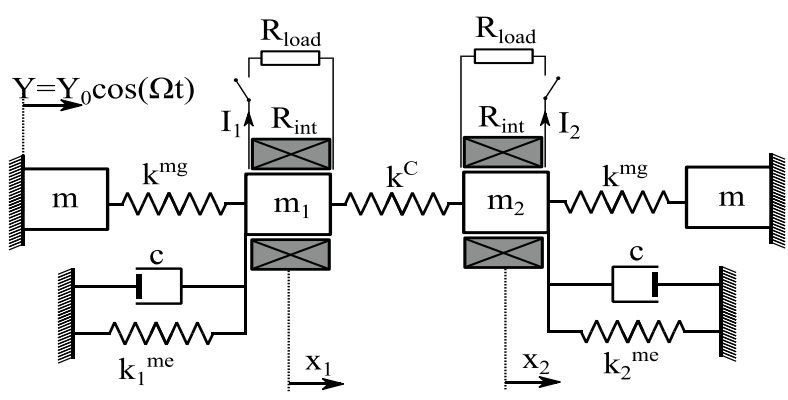

Fig. 1. Equivalent electrical and mechanical model for the proposed VEH device.

It is assumed that the two middle moving magnets have the same mass, size, and the identical mechanical and electrical damping.

The mechanical force, describes the behaviour of the used elastic spring, was modelled by Ansys software in the static model with considering the geometrical nonlinearity.

Figure 2 illustrates the numerical results obtained for the used elastic spring in function of the relative displacement. The obtained results were fitted in order to determine the linear and cubic nonlinear stiffness coefficients $k_{1}^{m e}$ and $k_{3}^{m e}$. As shown in this figure, the numerical and fitted results are in good agreement. Considering the proposed design, the maximal amplitude cannot exceed $5 \mathrm{~mm}$. In this sense, the linear expression can describe the behaviour of the elastic spring. Therefore, the cubic nonlinear stiffness $k_{3}^{m e}$ is neglected.

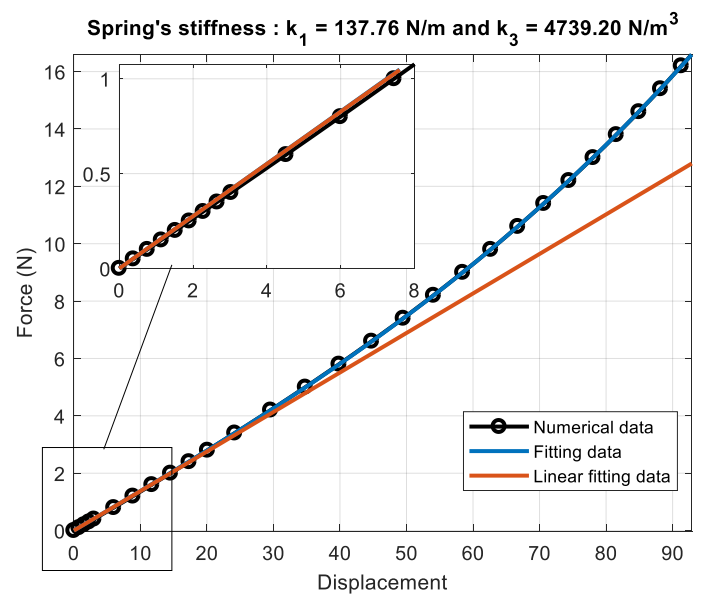

Fig. 2. Mechanical force with respect to the relative displacement.

On the other hand, the repulsive magnetic force has been estimated by the semi-analytical method called the equivalent surface current model (ESCM).

$$
\begin{aligned}
F\left(d_{0}\right) & =\mu_{0} \pi\left(R M_{s}\right)^{2} \int_{0}^{\infty} J_{1}(\varepsilon R)^{2}\left[2 e^{-\varepsilon\left(d_{0}+h_{m a g}\right)}\right. \\
& \left.-e^{-\varepsilon \cdot g a p}-e^{-\varepsilon\left(d_{0}+2 h_{m a g}\right)}\right] \varepsilon^{-1} d \varepsilon,
\end{aligned}
$$

where $J_{1}(\varepsilon R)$ is the $1^{\text {st }}$ order Bessel function. $\mu_{0}=$ $4 \pi 10^{-7}$ H.m ${ }^{-1}$ stands for the vacuum magnetic permeability. $M_{S}$ is the saturation magnetization of the identical magnets. $R$ and $h_{m a g}$ are the radius and height, of the magnets.

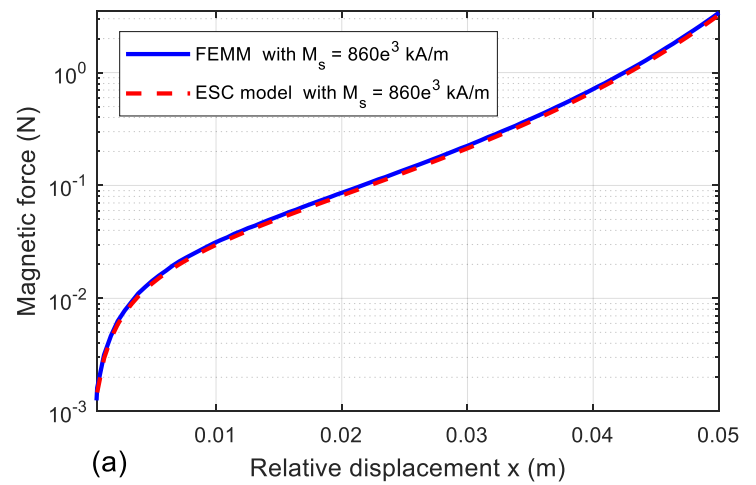

Fig. 3. Comparison of the magnetic force estimated by ESCM and FEMM with $d_{0}=60 \mathrm{~mm}$.

The accuracy of the results obtained by the ESC model was checked with the open numerical software FEMM [11]. As shown in Figure 3, the both models are in good agreement. The obtained results of the magnetic force estimated by ESCM have been fitted at different values of the gap $d_{0}$ and the relative displacement $x$ using a leastsquares procedure. So, the total magnetic force can be identified as:

$$
F_{j}^{m g}(x)=k_{1}^{m g} x+k_{3}^{m g} x^{3}
$$

where $k_{1}^{m g}=2 \lambda_{1}+4 d_{0} \lambda_{2}+6 d_{0}^{2} \lambda_{3}$ is the linear stiffness coefficient and $k_{3}^{m g}=2 \alpha_{3}$ is the cubic nonlinear stiffness coefficient in which $d_{0}$ is the gap between the magnets. Figure 4 exhibits the fitted data of the total magnetic force with respect to the relative displacement. The fitted data can describe the total magnetic force up to $30 \%$ of the gap compared to the ESC model.

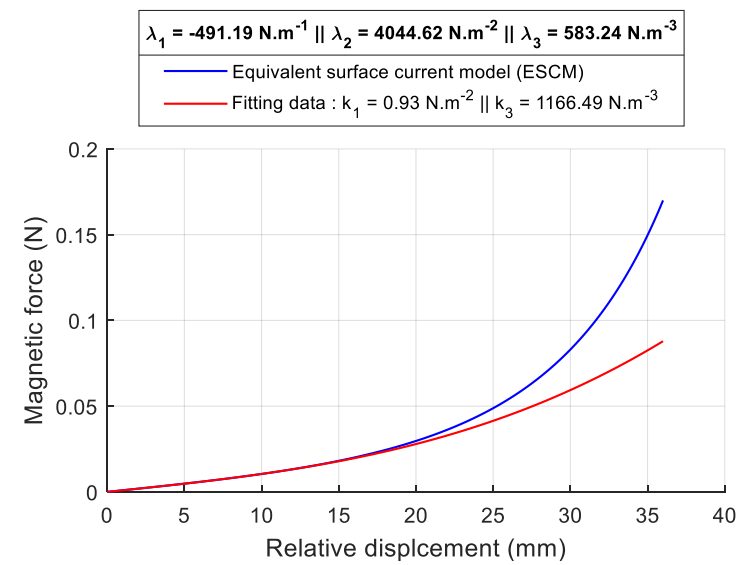

Fig. 4. Magnetic force with respect to the relative displacement with $d_{0}=60 \mathrm{~mm}$.

The estimated parameters for the magnetic linear stiffness at $d_{0}=60 \mathrm{~mm}$ are $\lambda_{1}=-491.19 \mathrm{~N} . \mathrm{m}^{-1}, \quad \lambda_{2}=$ $4044.62 \mathrm{~N} . \mathrm{m}^{-2}$, and $\lambda_{3}=583.24 \mathrm{~N} . \mathrm{m}^{-3}$. 
The governing equations of the proposed VEH device become as follows by substituting the expression of the magnetic and mechanical forces into equation 1 :

$$
\left\{\begin{array}{c}
\ddot{x}_{1}+2\left(\xi_{e}+\xi_{m}\right) \omega_{1} \dot{x}_{1}+\omega_{1}^{2}(1+2 \beta) x_{1}-\beta x_{2} \\
-\beta_{N L} x_{2}^{3}=-\ddot{Y} \\
\alpha \ddot{x}_{2}+2\left(\xi_{e}+\xi_{m}\right) \omega_{1} \dot{x}_{2}+\omega_{1}^{2}(1+2 \beta) x_{2}-\beta x_{1} \\
-\beta_{N L} x_{1}^{3}=-\alpha \ddot{Y}
\end{array}\right.
$$

where $2 \xi m_{1} \omega_{1}=c, \beta=\frac{k_{c}^{L}}{k_{1}^{m e}}, \beta_{N L}=\frac{k_{c}^{N L}}{m_{1}}, \omega_{1}^{2}=\frac{k_{1}^{m e}}{m_{1}}, \alpha=$ $\frac{m_{2}}{m_{1}}, . \alpha$ and $\beta$ are the mass mistuning and coupling coefficients, respectively.

The solving procedure uses the classical harmonic balance method combined with the asymptotic numerical method [12]. This technique allows transforming the nonlinearity present in the governing equation (equation 4 ) into purely polynomial quadratic terms.

The magnetic field $B$ of the permanent magnets has been obtained analytically by the expression developed for cylindrical magnets [13]:

$$
B(x)=\frac{\mu_{0} M_{S}}{2}\left(\frac{x}{\sqrt{x^{2}+R^{2}}}-\frac{x-h_{m a g}}{\sqrt{\left(x-h_{m a g}\right)^{2}+R^{2}}}\right)
$$

where $x$ is the relative displacement and $R$ is the radius of the moving magnets. The electromagnetic coupling $\gamma$ was estimated by the following expression [14]:

$$
\gamma=2 N \pi R \int \frac{B\left(x, h_{m a g}, R\right)}{d x} d h
$$

where $N=70$ is the number of winding of the coil. The calculated electromagnetic coupling $\gamma=1.019$ V.s. $\mathrm{m}^{-1}$ has been compared to the one $\gamma=1.089$ V.s. $m^{-1}$ estimated experimentally by measuring the output voltage at different load resistance values $R_{\text {load }}$.

The average of the total harvested power can be expressed by the following formula:

$$
P_{\text {avg }}=\left(\frac{\gamma}{\sqrt{2}\left(R_{\text {load }}+R_{\text {int }}\right)}\right)^{2} R_{\text {load }}\left(\dot{x}_{1}^{2}+\dot{x}_{2}^{2}\right)
$$

where $R_{\text {load }}$ and $R_{\text {int }}$ are the load and inner resistances. $\dot{x}_{1}$ and $\dot{x}_{2}$ are the velocities of the first and second dofs.

\section{RESULTS AND DISCUSSION}

In the present section, several numerical simulations have been performed in the case of two moving magnets. These simulations enable us to highlight the benefits of the energy localization phenomenon on the proposed harvesting device.

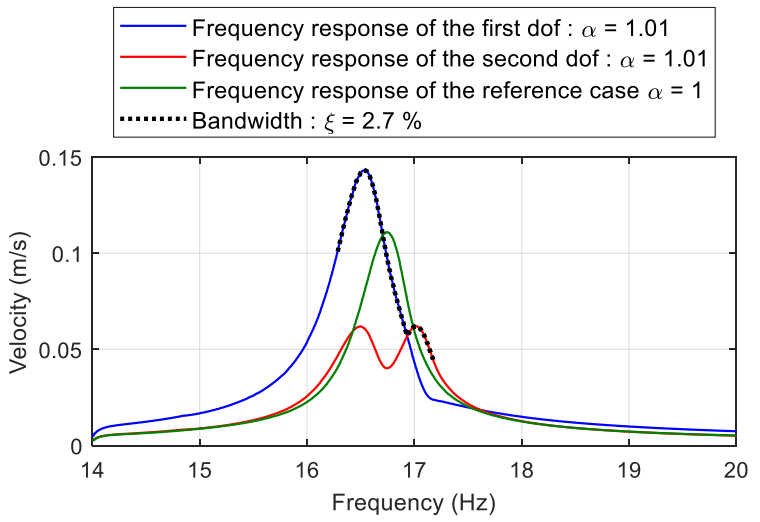

Fig. 5. Frequency response of the proposed harvester with $d_{0}=$ $60 \mathrm{~mm}$.

Figure 5 exhibits the frequency response of the proposed VEH device. This later shows an increasing of the velocity of the perturbed dof by approximately $21 \%$. Also, the effective bandwidth of the VEH device was enlarged by $14 \%$ compared to the reference case.

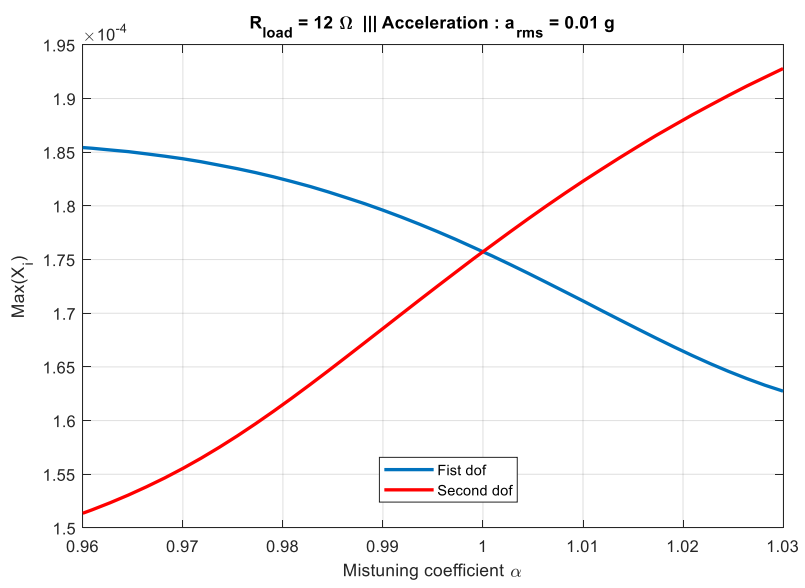

Fig. 6. Maximal oscillation amplitude at different mass mistuning coefficient values $\alpha$ with $d_{0}=60 \mathrm{~mm}$.

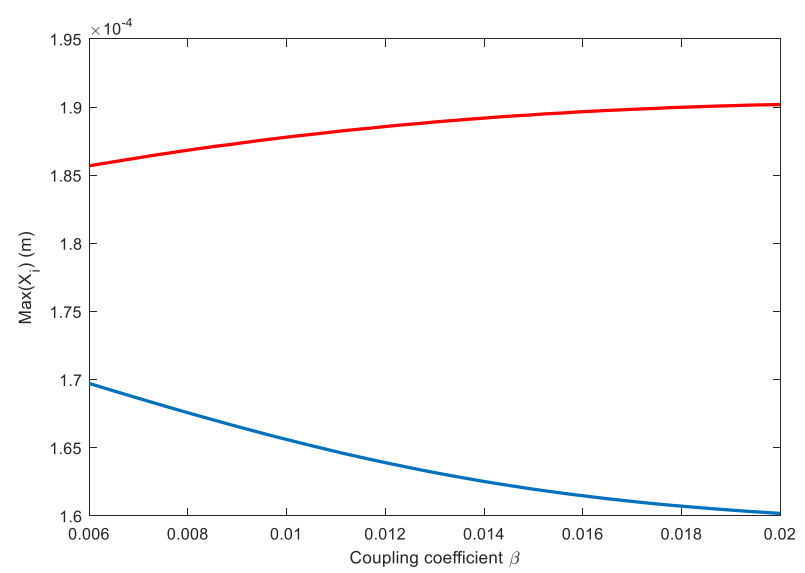

Fig. 7. Maximal oscillation amplitude at different magnetic coupling values $\beta$ with $d_{0}=60 \mathrm{~mm}$.

Figure 6 and 7 represent the variation of the maximal oscillation amplitudes at different values of the mass mistuning and magnetic coupling coefficients $\alpha$ and $\beta$. It can be noticed in Figure 6 that an increasing of the oscillation amplitudes of the proposed VEH device 
compared the case with $\alpha=1$. In addition, the magnetic coupling coefficient $\beta$ shows an effect on the oscillation amplitudes as shown in Figure 7.

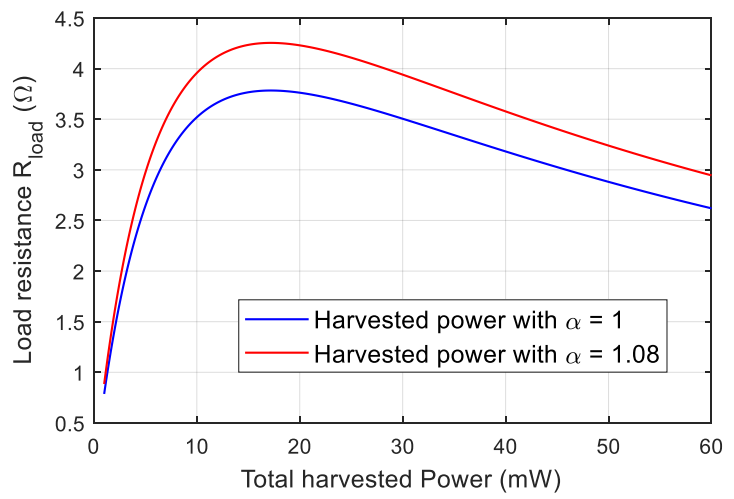

Fig. 7. Total harvested power for $\alpha=1$ and 1.08 with $d_{0}=$ $60 \mathrm{~mm}$.

Figure 7 exhibits the maximal powers at different load resistance with a gap $d_{0}=60 \mathrm{~mm}$ harvested from the proposed VEH device in two cases $\alpha=1$ and 1.08 . The estimated magnetic coupling for this gap is $\beta=0.67 \%$. In the first case, the maximal power has been harvested from the both dofs without any perturbation $\alpha=1$ while in the second case the proposed VEH device was perturbed $\alpha=1.08$. As shown in this Figure, the harvested maximal power with $\alpha=1.08$ was increased approximately $11 \%$ compared to the first case with $\alpha=$ 1.

\section{CONCLUSIONS}

In the present work, the effect of the energy localization phenomenon and the magnetic coupling on the frequency response of a periodic structure have been studied. The obtained results show that the perturbation of one of the moving magnet and the magnetic coupling coefficient $\beta$ increase the oscillation amplitude of the periodic system. Thus, we can take advantage of these aspects to enhance the harvested power of a vibration energy harvesting mechanism. As mentioned before, the total harvested power was improved approximately by $11 \%$ compared to the case without any perturbation. Also, thanks to the energy localization phenomenon, the effective bandwidth was enlarged by $14 \%$.

\section{ACKNOWLEDGEMENTS}

This project has been performed in cooperation with the Labex ACTION program (contract ANR-11-LABX-01-01).

\section{References}

1. C. Wei and X. Jing, Renewable and Sustainable Energy Reviews 74, 1 (2017).

2. N. Tran, M. H. Ghayesh, and M. Arjomandi, International Journal of Engineering Science, 127, 162-185 (2018).
3. A. Toprak and O. Tigli, Applied Physics Reviews 1, 031104 (2014).

4. S. Priya, H. C. Song, Y. Zhou, R. Varghese, A. Chopra, S. G. Kim, I. Kanno, L. Wu, D. S. Ha, J. Ryu, and R. G. Polcawich, Energy Harvesting and Systems 4, 3 (2017).

5. Z. Wu, J. Tang, X. Zhang, and Z. Yu, Applied Physics Letters 111, 013903 (2017).

6. S. Dirk and M. Yiannos, Electromagnetic Vibration Energy Harvesting Devices (Springer, 2012).

7. Y. Zhang, T. Wang, A. Zhang, Z. Peng, D. Luo, R. Chen, and F. Wang, Review of Scientific Instruments 87, 125001 (2016).

8. S. Mahmoudi, N. Kacem and N. Bouhaddi, Enhancement of the performance of a hybrid nonlinear vibration energy harvester based on piezoelectric and electromagnetic transductions, Smart Materials and Structures 23, 075024 (2014).

9. I. Abed, N. Kacem, N. Bouhaddi and M. L. Bouazizi, Multi-modal vibration energy harvesting approach based on nonlinear oscillator arrays under magnetic levitation. Smart Materials and Structures, 25, 025018 (2016).

10. C. Drezet, N. Kacem and N. Bouhaddi, Design of a nonlinear energy harvester based on high static low dynamic stiffness for low frequency random vibrations, Sensors and Actuators A: Physical (2018).

11. D. C. Meeker, http://www.femm.inf (2006).

12. B. Cochelin and C. Vergez, Journal of Sound and Vibration, 324, 243-262 (2009).

13. J. M. Camacho, V. Sosa, Revista Mexicana de Fisica, 59, 8-17 (2013).

14. S. Dirk and M. Yiannos, Electromagnetic Vibration Energy Harvesting Devices, Springer (2012). 